\title{
Demagnetizing effects in stacked rectangular prisms
}

Christensen, Dennis; Nielsen, Kaspar Kirstein; Bahl, Christian Robert Haffenden; Smith, Anders

Published in:

Journal of Physics D: Applied Physics

Link to article, DOI:

$10.1088 / 0022-3727 / 44 / 21 / 215004$

Publication date:

2011

Link back to DTU Orbit

Citation (APA):

Christensen, D., Nielsen, K. K., Bahl, C. R. H., \& Smith, A. (2011). Demagnetizing effects in stacked rectangular prisms. Journal of Physics D: Applied Physics, 44(21), 215004. https://doi.org/10.1088/0022-3727/44/21/215004

\section{General rights}

Copyright and moral rights for the publications made accessible in the public portal are retained by the authors and/or other copyright owners and it is a condition of accessing publications that users recognise and abide by the legal requirements associated with these rights.

- Users may download and print one copy of any publication from the public portal for the purpose of private study or research.

- You may not further distribute the material or use it for any profit-making activity or commercial gain

- You may freely distribute the URL identifying the publication in the public portal

If you believe that this document breaches copyright please contact us providing details, and we will remove access to the work immediately and investigate your claim. 


\title{
Demagnetizing effects in stacked rectangular prisms
}

\author{
D.V. Christensen ${ }^{1,2}$, K.K. Nielsen ${ }^{1}$, C.R.H. Bahl ${ }^{1}$ and A. \\ Smith $^{1}$ \\ ${ }^{1}$ Fuel Cells and Solid State Chemistry Division Ris $\varnothing$ National Laboratory for \\ Sustainable Energy, Technical University of Denmark, Frederiksborgvej 399, DK-4000 \\ Roskilde, Denmark \\ 2 The Niels Bohr Institute, University of Copenhagen, Blegdamsvej 17, DK-2100 \\ Copenhagen, Denmark \\ E-mail: dechr@risoe.dtu.dk
}

\begin{abstract}
A numerical, magnetostatic model of the internal magnetic field of a rectangular prism is extended to the case of a stack of rectangular prisms. The model enables the calculation of the spatially resolved, three-dimensional internal field in such a stack given any magnetic state function, stack configuration, temperature distribution and applied magnetic field. In this paper the model is applied to the case of a stack of parallel, ferromagnetic rectangular prisms and the resulting internal field is found as a function of the orientation of the applied field, the number of prisms in the stack, the spacing between the prisms and the packing density of the stack. The results show that the resulting internal field is far from being equal to the applied field and that the various stack configurations investigated affect the resulting internal field significantly and non-linearly. The results have a direct impact on the design of, e.g., active magnetic regenerators made of stacked rectangular prisms in terms of optimizing the internal field.
\end{abstract}

PACS numbers: 75.30.Sg,75.30.-m,75.60.Ej,41.20.Gz 


\section{Introduction}

The total magnetic field, $\mathbf{H}$, in the vicinity of one or several magnetized bodies is of general interest. In particular, when concerned with, e.g., magnetic refrigeration the local magnetic field of the magnetic material is of great importance $[1,2]$. The magnetostatic calculation of $\mathbf{H}$ is in principle straightforward and can in certain cases, e.g. ellipsoids, be found analytically [3, 4, 5]. However, this is only true for homogeneously magnetized bodies. When the magnetization of the body is varying spatially, numerical methods are usually required $[1,6,7,8]$.

In the presence of magnetized bodies the total magnetic field at a certain point in space, $\mathbf{r}$, can be found as the superposition of the applied magnetic field, $\mathbf{H}_{\text {appl }}$, and the magnetic field created by the magnetized bodies. The magnetic field from a single, magnetic body is typically called the demagnetizing field inside the body and the stray or interaction field outside. Since the source of these fields is the same, the remainder of this paper will adopt the concept of the magnetic field of the body, $\mathbf{H}_{\text {body }}(\mathbf{r})$, at any given point in space. The resulting total magnetic field may thus be written as

$$
\mathbf{H}(\mathbf{r})=\mathbf{H}_{\text {appl }}(\mathbf{r})+\sum_{i=1}^{N} \mathbf{H}_{\text {body }, \mathrm{i}}(\mathbf{r}),
$$

where the index $i$ represents the $i$ th body out of a total of $N$ bodies. Usually, the solution to the magnetic field from a single, homogeneously magnetized body with magnetization $\mathbf{M}$ is written as

$$
\mathbf{H}_{\text {body }}(\mathbf{r})=-\mathbb{N}(\mathbf{r}) \cdot \mathbf{M}
$$

where the demagnetizing tensor field, $\mathbb{N}(\mathbf{r})$, only depends on the geometry of the body.

Under inhomogeneous conditions iterative methods are typically required to determine the internal magnetic field of a magnetized body $[4,6]$. Furthermore, even in a homogeneous applied magnetic field, the field of a magnetized body is generally inhomogeneous. In non-saturated conditions the magnetization thus becomes a nontrivial function of position thereby making evaluation of the magnetic field due to the magnetization of the body possible only using numerical methods.

Here, the numerical solution to the total magnetic field of a configuration of multiple bodies with inhomogeneous and field-dependent magnetization is reported. The numerical model is applied to a stack of rectangular prisms as this configuration is important in, e.g., magnetic refrigeration [9]. However, the discretization underpinning the model can in principle be applied to any shape. The implementation is described in Sec. 2 and the results of different stack configurations are presented and discussed in Sec. 3. In Sec. 4 the implications of the model are considered. Finally, in Sec. 5, the conclusions are provided. 


\section{Magnetostatic model of stacked rectangular prisms}

In the following a numerical model capable of calculating the total magnetic field in a configuration of $N$ stacked rectangular prisms, as depicted in Fig. 1, is presented. The model is an extension of the single prism solution, which was presented in Ref. [8] and experimentally verified in Ref. [10]. The single prism model uses iteration to solve the two coupled equations

$$
\mathbf{M}(\mathbf{r})= \begin{cases}f(T(\mathbf{r}), H(\mathbf{r})) \frac{\mathbf{H}(\mathbf{r})}{H(\mathbf{r})} & \text { inside the prism } \\ 0 & \text { outside the prism }\end{cases}
$$

and

$$
\mathbf{H}(\mathbf{r})=\mathbf{H}_{\text {appl }}(\mathbf{r})+\mathbf{H}_{\text {body }}(\mathbf{r}) .
$$

Here $f$ gives the magnitude of the local magnetization of the prism as a function of the (spatially dependent) temperature, $T$, and internal field strength, $H$; $\mathrm{f}$ is determined by the magnetic equation of state. Note that the magnetization is taken to be along the direction of the internal magnetic field thereby assuming the material to be isotropic. To solve Eqs. 3-4 iteratively, expressions for $f$ and the magnetic field produced by the magnetic body, $\mathbf{H}_{\text {body }}$, need to be established.

The magnetic equation of state is chosen to be the mean field equation of state [11] with parameters appropriate for the rare earth metal gadolinium (see Ref. [8]) frequently used in magnetic refrigeration. The temperature is chosen to be $293 \mathrm{~K}$, which is also the Curie temperature of gadolinium. The model may straightforwardly be extended to handle temperature and material variations across the prisms, however, for simplicity this is not done in the present study; see Ref. [8] for further details.

The magnetic field of a single magnetized rectangular prism is found by dividing the prism into $k$ cells each shaped as a rectangular prism small enough to be approximately homogeneously magnetized, whereas both the magnitude and direction of the magnetization may vary from cell to cell. The magnetic field of each homogeneously magnetized cell can be expressed analytically [4] and hence the total magnetic field due to the magnetization can be obtained by superimposing the contributions from each cell

$$
\mathbf{H}_{\text {body }}(\mathbf{r}) \approx-\sum_{j=1}^{k} \mathbb{N}\left(\mathbf{r}-\mathbf{r}_{j}\right) \cdot \mathbf{M}_{j}
$$

where $\mathbf{r}_{j}$ denotes the center of the $j$ th cell, $\mathbf{M}_{j}$ is the magnetization of the $j$ th cell and $\mathbb{N}$ is the symmetric $3 \times 3$ tensor field with components given in Ref. [8]. Note that the expression given in Eq. 5 is an approximation that relies on the discretization of the rectangular prism into small cells; see Ref. [8] for details on sufficient grid size and the numerical implementation.

The extension to a configuration of multiple prisms is done by extending the sum in Eq. 5 to include each individual prism, i.e. by combining Eqs. 1 and 5. The total 


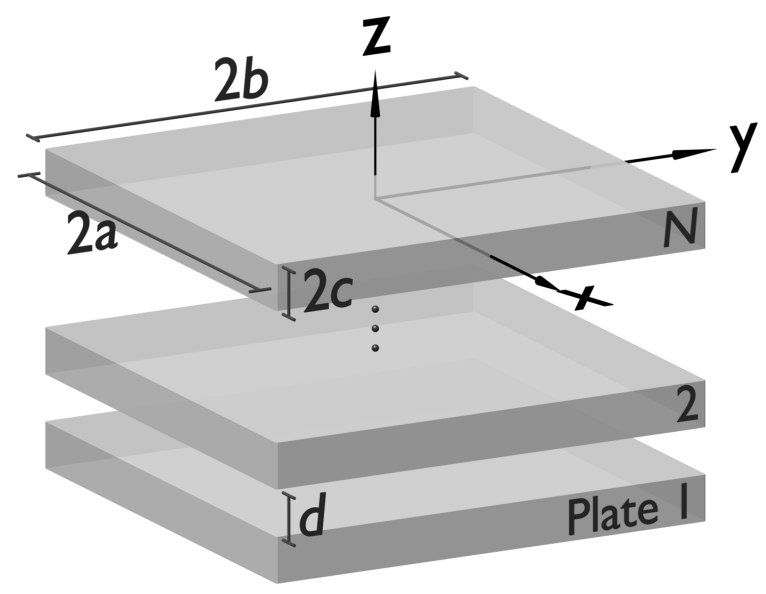

Figure 1. The coordinate system of a stack composed of rectangular prisms each with dimensions $2 a \times 2 b \times 2 c$. The stacking of the prisms is in the $z$-direction where the prisms are thinnest. The total height of the stack is denoted $L$ and the distance between two adjacent prisms is $d$.

magnetic field therefore becomes

$$
\mathbf{H}(\mathbf{r}) \approx \mathbf{H}_{\mathrm{appl}}(\mathbf{r})-\sum_{i=1}^{N} \sum_{j=1}^{k} \mathbb{N}\left(\mathbf{r}-\mathbf{r}_{i, j}\right) \cdot \mathbf{M}_{i, j},
$$

where $N$ is the number of prisms and $i$ is used to index the prisms. Thus, the extension to multiple prisms is obtained by dividing each prism into a set of cells and superimposing the contributions from all cells. Note that each step of the iteration ranges over the entire array, not just over a single prism. The present approach can readily be extended to cover the case of a two- or three-dimensional array of rectangular prisms. In addition, any other collection of arbitrarily shaped bodies can be divided into cells and solved using the method described here.

\section{Results and discussion}

The model is applied to the case of $N$ prisms equally spaced with a distance $d$ between adjacent prisms resulting in a spatial extent $L=N 2 c+(N-1) d$ of the stack in the $z$-direction as seen in Fig. 1. Following Ref. [8] the dimensions of each prism have been chosen to be $2 a \times 2 b \times 2 c=20 \times 20 \times 1 \mathrm{~mm}^{3}$ and an applied magnetic field of 1 $\mathrm{T}$ is oriented along either the $x$ - or the $z$-direction. Three sets of parameter variations are considered. Firstly, the number of prisms in the stack is varied while the distance between two adjacent prisms is kept constant. Secondly, a stack of a fixed number of prisms is considered in which the distance between the prisms is varied. Thirdly, the overall spatial extent of the stack in the direction of the stacking is kept constant (i.e. $L$ is kept fixed). The number of prisms, the distance and the thickness of the prisms are then varied within this space in order to probe the effect of the packing density of the stack on the internal magnetic field of the stack. 


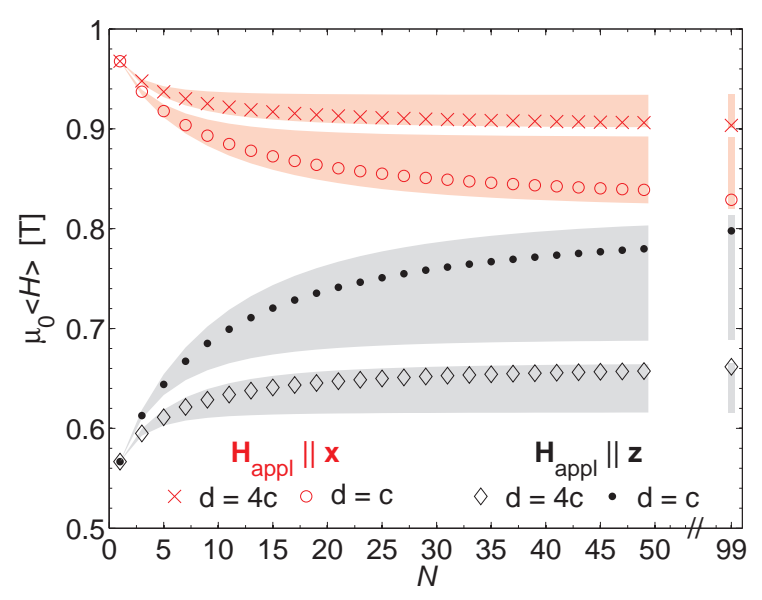

Figure 2. The average internal magnetic field strength in stacks composed of $N$ prisms. The prisms are stacked with a distance of $d=c$ or $d=4 c$ and subjected to two orientations of the applied magnetic field. The shaded areas cover the area between the lowest and highest values of the average internal field in the individual prisms.

\subsection{Varying the number of prisms}

The number of prisms in the stack, $N$, is varied from 1 to 99 and the spacing between two adjacent prisms is kept fixed at $d=c$ or $d=4 c$. In the former case the spacing between two adjacent prisms is equal to half the thickness of a single prism whereas in the latter it is equal to the thickness of two prisms. These two cases result in packing densities of $2 / 3$ and $1 / 3$, respectively, where the packing density, $\alpha$, is given by

$$
\alpha=\frac{2 c}{d+2 c} \text {. }
$$

The volume average of the internal magnetic field strength in all the prisms is given in Fig. 2 for an applied magnetic field oriented along either the $x$ - or $z$-direction. For a single prism the volume average of the internal magnetic field strength attains its lowest value when the applied field is along the $z$-direction as the demagnetizing field is maximized for this orientation. The opposite is true when the applied field is in the $x$-direction. These results follow from the well-known single prism solution that may be found in, e.g., Ref. [5].

Increasing the number of prisms when the applied magnetic field is perpendicular to the direction of the stacking (i.e. $\mathbf{H}_{\text {appl }} \| \mathbf{x}$ ) reduces the total average internal field of the stack. Conversely, the total average internal field increases when the applied field is along the stacking direction $\left(\mathbf{H}_{\text {appl }} \| \mathbf{z}\right)$. This is to be expected and simply explained by considering Fig. 3. When the stacking direction is parallel with the applied field the stray field due to the magnetization of each individual magnetized body will tend to enhance the applied field in neighboring prisms, whereas the stray field tends to reduce it when the stacking is perpendicular to the applied field. Note that this entails that the largest internal field is experienced by the center prism for $\mathbf{H}_{\text {appl }} \| \mathbf{z}$ and the outermost 


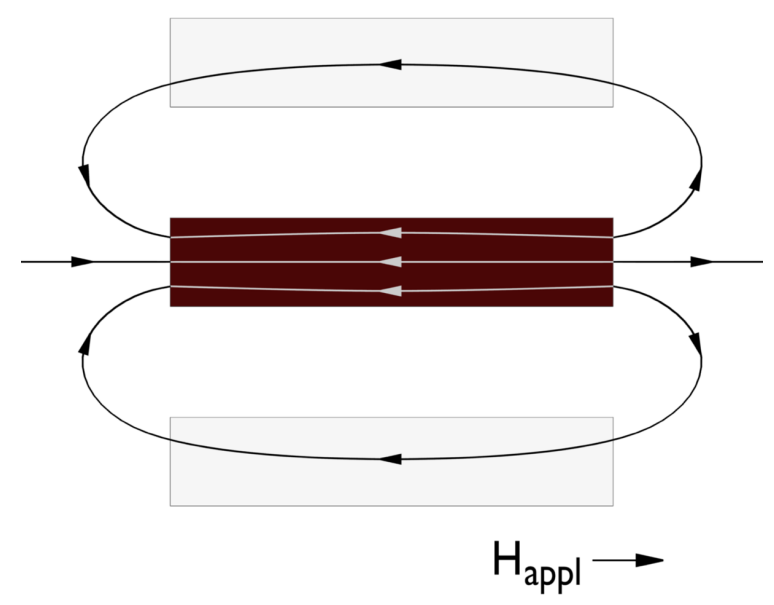

(a)

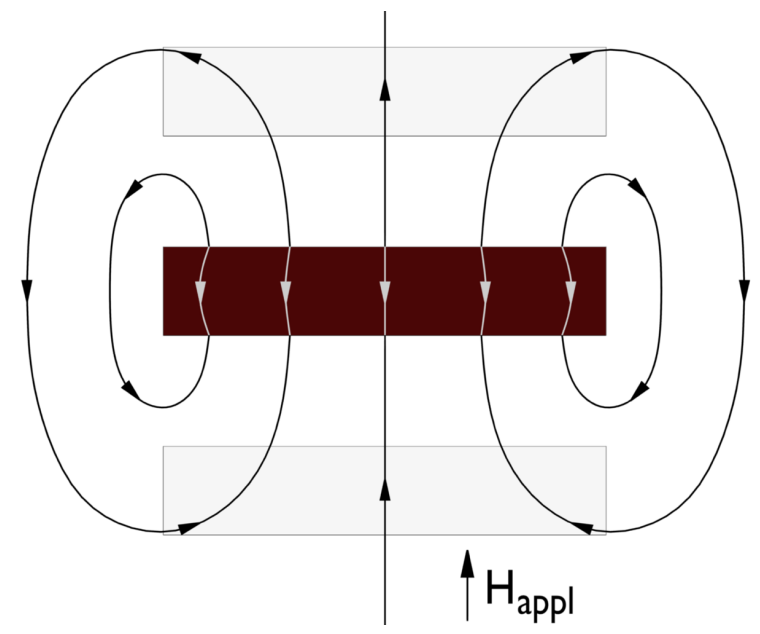

(b)

Figure 3. Schematic illustration of the magnetic field from a single rectangular prism positioned in a stack. The prism is magnetized either perpendicular to the stacking direction (a) or parallel (b). In both cases the magnetic field from the prism opposes the applied field inside the prism as seen by the white field lines. Stacking the prisms perpendicular to the magnetization direction results in a decrease in the internal magnetic field, whereas parallel stacking enhances the internal field. The trends are similar for both a homogeneously magnetized prism and the inhomogeneously magnetized prism considered here. Note that the internal magnetic field is discontinuous across the surfaces containing effective magnetic charges as expected from the boundary conditions of the $\mathbf{H}$-field.

prisms for $\mathbf{H}_{\text {appl }} \| \mathbf{x}$, whereas the minimum internal field is found at the outer prisms and the center prism for $\mathbf{H}_{\text {appl }} \| \mathbf{z}$ and $\mathbf{H}_{\text {appl }} \| \mathbf{x}$, respectively. The minimum and maximum values for the individual prisms are represented by the shaded areas in Fig. 2 .

As the number of magnetized bodies increases, the field enhancing or reducing effect is enlarged steadily, reaching a level where the stack of prisms behaves as an infinite stack. In this case the outer boundaries become virtually negligible. This is clearly apparent from Fig. 2 for $d=4 c$ where increasing the number of prisms from $N=49$ to 99 only changes the average internal field by $0.3 \%$ and $0.6 \%$ when the applied field is along the $x$ - and $z$-direction, respectively. In addition the average internal field of the whole stack almost coincides with the average internal field of the center prism thereby indicating that the boundaries are indeed of minor importance. In the case where $d=c$ the average of the total internal field in the stack is also seen to behave asymptotically, however, the convergence is less pronounced. A better description of the infinite stack is found by only considering the center prism instead as convergence is essentially reached at $N=99$ and consequently the influence of the boundaries are negligible. In the remainder of this paper the internal field of the center prism in a stack of 99 prisms will therefore be used when referring to an infinite stack. Note that this assumption improves with increasing distance between the prisms. 


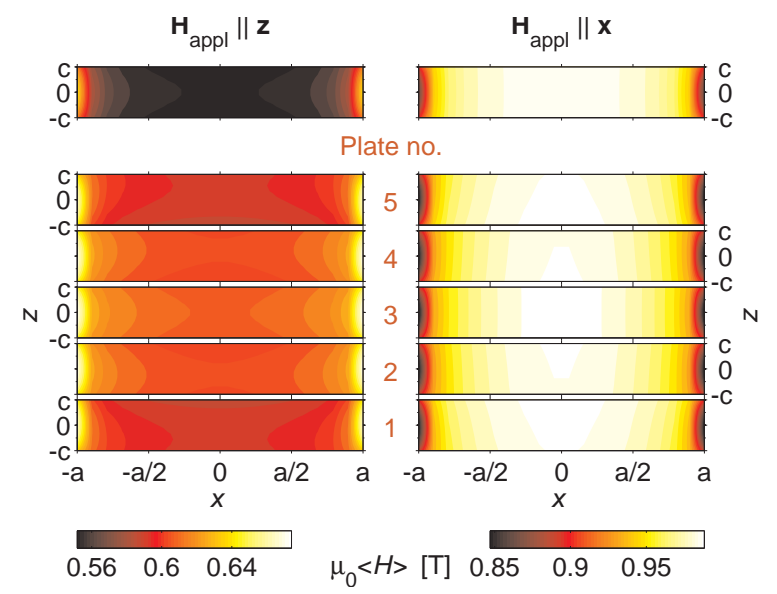

Figure 4. The internal magnetic field strength of a single rectangular prism (top) and a configuration of 5 prisms displaced with $d=4 c$ (bottom; not drawn to scale) for two different orientations of the applied magnetic field. The internal magnetic field strength is averaged in the $y$-direction.

Figure 4 shows an example of the spatial variations in the magnitude of the internal field in a single prism and a stack of five prisms spaced with $d=4 c$. It is observed that the internal field is significantly affected when the number of prisms in the stack is greater than one. The difference between a single prism and the stack of five prisms is most pronounced in the case where the applied field is along the $z$-direction. In addition, the internal field in the middle prism is seen to differ from that of the surrounding prisms. This implies that the magnetization also differs and consequently all prisms must be included in the iteration performed in Eqs. 3-4 rather than using a sum of single-prism solutions.

\subsection{Varying the distance between the prisms}

For an infinite stack and a stack containing 19 prisms the average internal magnetic field strength is plotted in Fig. 5 as a function of the distance between the prisms given in units of the prism thickness, $d / 2 c$. As in the previous section the shaded areas in Fig. 5 mark the maximum and minimum values of the average internal field strength in the individual prisms for a given stack configuration. An increase in the distance is observed to result in an asymptotical approach towards a value of 0.96 and $0.57 \mathrm{~T}$ for an applied field along the $x$ - and $z$-direction, respectively. This behavior is almost identical for the two stacks considered. When the distance between adjacent prisms increases the stray field from each magnetized prism has less influence on neighboring prisms and the number of prisms in the individual stacks becomes less important. The asymptotic values are therefore equal to the single prism solutions seen in Fig. 2.

Interestingly, the results from the two stack configurations differ considerably at low values of the ratio $d / 2 c$. Here, the stray fields from the magnetized prisms have a much larger influence on neighboring prisms, as expected, which is also clearly manifested in a great response of the internal magnetic field strength when varying the distance. In 


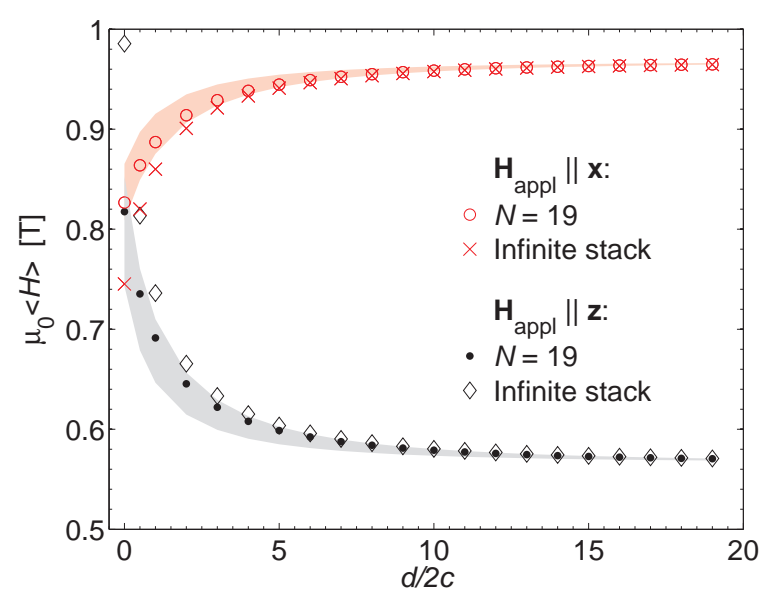

Figure 5. The average internal field strength in stacks composed of rectangular prisms as a function of the distance between two adjacent prisms normalized to the thickness of a single prism. Two orientations of the applied field are provided (along the $x$ - and the $z$-direction). Furthermore, an infinite stack and a stack composed of 19 prisms are considered. As in Fig. 2 the shaded areas mark the maximum and minimum average internal field strengths of the individual prisms in the given configuration.

addition, when the applied magnetic field is along the $z$-axis and $d / 2 c$ is close to zero the average internal field strength is actually significantly larger than in the case where the applied field is along the $x$-direction. This is not surprising considering the limit where $d / 2 c$ is zero since the stack is then effectively a single prism with dimensions $2 a \times 2 b \times 2 c N$; here, the single prism solution dictates that the average internal field should be greater when applying the external magnetic field along the direction where the prism is thickest [5]. In particular for the infinite stack the magnetic field from the magnetization vanishes if $d / 2 c=0$ and the applied field is oriented along the direction of stacking. This is observed in Fig. 2 by the strength of the internal magnetic field almost being equal to the applied field $(1 \mathrm{~T})$. The small discrepancy is solely due to the fact that the infinite stack is represented by a prism surrounded by a large, albeit finite number of prisms.

\subsection{Varying the packing density}

Recalling the definition of the packing density, Eq. 7, maintaining a constant total stack height, $L$, and adding prisms such that the distance between adjacent prisms for a given number of prisms is constant, the packing density of the stack may be varied consistently. In addition the packing density can be varied by changing the thickness of the prisms along the direction of stacking.

In Fig. 6a the average internal field strength of the stack is given as a function of the packing density for four different prism thicknesses. For all thicknesses it is observed that values of the packing density greater than about $90 \%$ yield the largest average internal field when the applied field is along the $z$-direction, whereas applying the field 


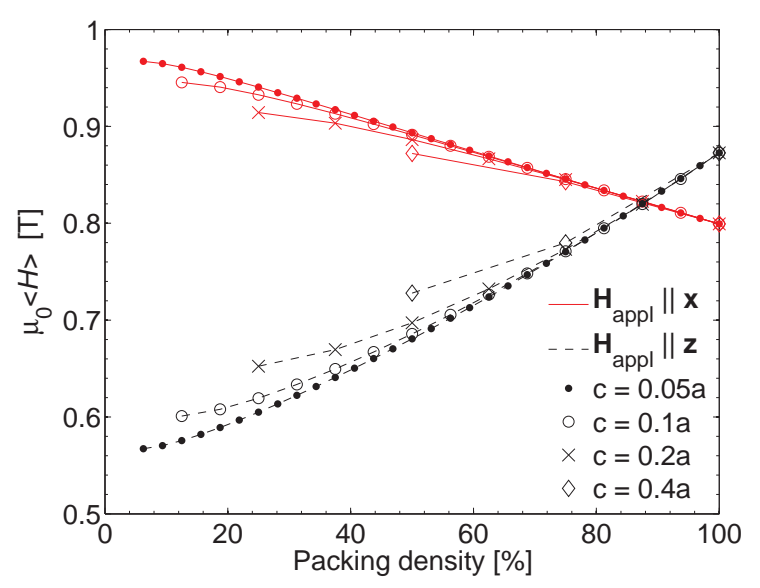

(a)

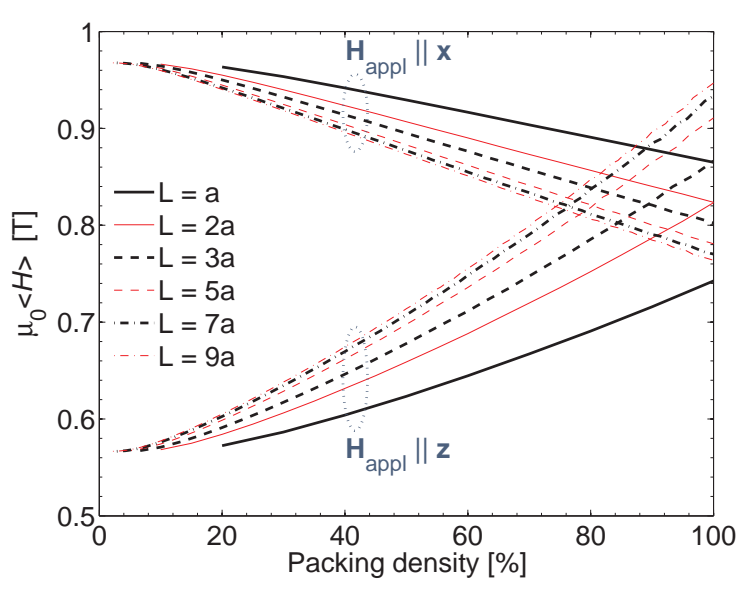

(b)

Figure 6. The average internal field strength in stacks composed of rectangular prisms as a function of the packing density. a) The packing density is varied by changing the number of equally spaced prisms while keeping the total height of the stack fixed at $L=3.2 a=32 \mathrm{~mm}$. Furthermore, four different prism thicknesses are provided, $c=0.05 a, c=0.1 a, c=0.2 a$ and $c=0.4 a$, and the applied field is oriented along either the $x$ - or the $z$-direction. b) The thickness of the prisms is kept fixed at $c=0.05 a$ whereas the total height of the stack is varied from $L=a$ to $L=7 a$. Note that for a packing density of one the stack becomes a single prism; in particular, when $L=2 a$ the stack is a cube.

in the $x$-direction results in the maximum average internal field of the stack for packing densities less than $90 \%$. As the packing density increases the stack approaches a single prism with dimensions $2 a \times 2 b \times L=20 \times 20 \times 32 \mathrm{~mm}^{3}$. Applying the field along the $z$-direction thus yields the strongest internal magnetic field in agreement with the single prism solution from Ref. [5]. On the other hand thin prisms located in a stack with a low packing density are almost isolated and the strongest internal field is therefore found when applying the external field along the $x$-direction in agreement with the results of, e.g., Fig. 2.

By comparing the results from different prism thicknesses the same trends are seen. When the packing density for a given prism thickness is increased the internal field of the stack is decreased for an applied field along the $x$-direction and increased when applying the field in the $z$-direction. The trends are due to the field reducing $\left(\mathbf{H}_{\text {appl }} \| \mathbf{x}\right)$ or enhancing $\left(\mathbf{H}_{\text {appl }} \| \mathbf{z}\right)$ effects of both adding more prisms and decreasing the distance between adjacent prisms. At any packing density the strongest internal field is found when the stack is composed of thin prisms for the case of an external magnetic field applied parallel to the $x$-direction, whereas thicker prisms result in the highest internal field when the applied field is along the $z$-direction.

The effect of varying the total height of the stack while maintaining a fixed thickness of the prisms is shown in Fig. 6b. Consistent with the single prism solution increasing the height of the stack for a packing density of one increases the internal magnetic if the applied magnetic field is along the $z$-direction and decreases the field for the applied 
field oriented along the $x$-direction. For a packing density of one and a stack height of $L=2 a$, the stack is a cube in which case applying the external field along any of the principal axes results in identical average internal fields. For smaller stack heights the largest internal field is obtained by applying the external magnetic field along the $x$-direction for all packing densities. At greater stack heights the optimal orientation of the applied field is less trivial as it is dependent on the actual stack configuration. For a stack height of, e.g., $L=3.2 a$ a value of around $90 \%$ was observed at which the applied field orientation that optimizes the internal field is changed. This value decreases non-linearly as the stack height is increased seemingly reaching a packing density of around $70 \%$ asymptotically. This limit can be deduced by noting that the stack in this case effectively becomes an infinite stack with an a priori unknown distance $d^{\prime}$ between neighboring prisms. Varying the packing density is thus equivalent to varying the distance between the prisms in an infinite stack. The distance $d^{\prime} \approx c$ can therefore be determined by reference to Fig. 5. Recalling the definition of the packing density, Eq. 7, the packing density at which the optimal direction of the applied field changes is approximately $2 / 3$.

\section{Implications of the model}

As we have seen, the magnetic field of the stack can differ significantly from the magnetic field of a single prism. This makes the optimization of the internal magnetic field nontrivial even for the simple stack configurations considered here. A direct implication of this is seen when considering magnetic refrigeration. Typically, stacks of parallel, ferromagnetic plates are used with the extent of the stack often determined by the spatial extent of the applied magnetic field. To increase heat transfer between the magnetic plates and the heat transfer fluid the plates are generally thin, whereas the number of plates is high in order to increase the volume of the active material. An important factor in determining the exact stack configuration and the orientation of the applied magnetic field is optimizing the internal magnetic field in the entire stack. However, considering Fig. 6a a large number of closely spaced, thin prisms results in significant magnetostatic interactions between the plates, making a numerical model a valuable tool for predicting and maximizing the internal magnetic field. In addition Fig. $6 \mathrm{~b}$ reveals that especially the packing density and the total height of the stack can highly influence the optimal orientation of the applied magnetic field. Extending the presented model to cover conditions relevant for magnetic refrigeration, such as a temperature gradient along the plates, which may be comprised of several materials, can readily be implemented by allowing temperature and material variations in the magnetic equation of state. For a single prism the implementation of these conditions has been reported previously [8], but in the light of the results presented here extending this to a stack of plates may yield an internal magnetic field significantly different from that of a single plate. In addition, applying the model to two- and three-dimensional arrays of rectangular prisms is likewise of great interest. 
Application of the presented model will also be relevant when considering electronic circuits where the local magnetic field play a significant role in certain cases $[14,15]$.

\section{Conclusion}

A numerical model capable of determining the spatially resolved, three-dimensional internal magnetic field in a configuration of multiple bodies with inhomogeneous and field-dependent magnetization was presented. The model was applied to a stack of equally spaced, rectangular prisms. The number of prisms, the separation between the prisms and the packing density were varied and the features of the resulting internal magnetic field explained qualitatively.

Generally, it is found that the stray field created by the magnetization of each prism can have a significant impact on the resulting internal magnetic field thus making it important to consider the full geometry of all magnetic bodies present to accurately describe the internal field. In particular it is concluded that the orientation of the applied field, which optimizes the internal field in the stack, may differ considerably from the optimal direction of the applied field for a single prism.

\section{Acknowledgements}

The authors thank the support of the Programme Commission on Energy and Environment (EnMi) (Contract no. 2104-06-0032), which is part of the Danish Council for Strategic Research.

\section{References}

[1] O. Peksoy and A. Rowe. Demagnetizing effects in active magnetic regenerators. Journal of Magnetism and Magnetic Materials, 288:424-432, 2005.

[2] R. Bjørk and K. Engelbrecht. The influence of the magnetic field on the performance of an active magnetic regenerator (AMR). International Journal of Refrigeration, Accepted, 2010.

[3] J.A. Osborn. Demagnetizing factors of the general ellipsoid. Physical Review, 67:351-357, 1945.

[4] R.I. Joseph and E. Schloemann. Demagnetizing field in nonellipsoidal bodies. Journal of Applied Physics, 36(5):1579-1593, 1965.

[5] A. Aharoni. Demagnetizing factors for rectangular ferromagnetic prisms. Journal of Applied Physics, 83(6):3432-3434, 1998.

[6] J.A. Brug and W.P. Wolf. Demagnetizing fields in magnetic measurements I. Thin discs. Journal of Applied Physics, 83(6):3432-3434, 1998.

[7] M. Beleggia and M. De Graef. On the computation of the demagnetization tensor field for an arbitrary particle shape using a fourier space approach. Journal of Magnetism and Magnetic Materials, 263:L1-L9, 2003.

[8] A. Smith, K.K. Nielsen, D.V. Christensen, C.R.H. Bahl, R. Bjørk, N. Pryds and J. Hattel. The demagnetizing field of a non-uniform rectangular prism. Journal of Applied Physics, 107(10):103910-1-8, 2010.

[9] C.R.H. Bahl, T.F. Petersen, N. Pryds and A. Smith. A versatile magnetic refrigeration test device. Review of Scientific Instruments, 79:093906-1-7, 2008. 
[10] D.V. Christensen, R. Bjørk, K.K. Nielsen, C.R.H. Bahl, A. Smith and S. Clausen. Spatially resolved measurement of the magnetocaloric effect and the local magnetic field using thermography. Journal of Applied Physics, 108:063913-1-4, 2010.

[11] A.H. Morrish. The Physical Priciples of Magnetism. John Wiley \& Sons, Inc., 1965.

[12] D.R. Lide. CRC Handbook of Chemistry and Physics. CRC Press, 2004.

[13] A.M. Tishin and Y.I. Spichkin. The Magnetocaloric Effect and its Applications. Institute of Physics Publishing, 2003.

[14] W. Andra, H. Danan and U. Ropke. Model for perpendicular hysteresis of thin magnetic films. IEEE Transactions on Magnetics, 20:102-104, 1984.

[15] A.N. Bogdanov, I.E. Dragunov and U.K. Rosz1er. Reorientation, multidomain states and domain walls in diluted magnetic semiconductors. Journal of Magnetism and Magnetic Materials, $316: 225-228,2007$.

[16] C.R.H. Bahl and K.K. Nielsen. The effect of demagnetization on the magnetocaloric properties of gadolinium. Journal of Applied Physics, 105:013916-1-5, 2009.

[17] K.K. Nielsen, R. Bjørk, J.B. Jensen, C.R.H. Bahl, N. Pryds, A. Smith, A. Nordentoft and J. Hattel. Magnetic cooling at Ris $\varnothing$. $8^{\text {th }}$ IIR Gustav Lorentzen Conference on Natural Working Fluids, Copenhagen, 2008.

[18] R. Bjørk, C.R.H. Bahl and M. Katter. Magnetocaloric properties of $\mathrm{LaFe}_{13-x-y} \mathrm{Co}_{x} \mathrm{Si}_{y}$ and commercial grade Gd. Journal of Magnetism and Magnetic Materials, 322:3882-3888, 2010.

[19] K.A. Gschneidner Jr., V.K. Pecharsky and A.O. Tsokol. Recent developments in magnetocaloric materials. Reports on Progress in Physics, 68:1479-1539, 2005.

[20] K.A. Gschneidner Jr. and V.K. Pecharsky Thirty years of near room temperature magnetic cooling: Where we are today and future prospects. International Journal of Refrigeration, 31:945-961, 2008. 